\title{
Research and implementation of integrated fingerprint classification system based on backtracking feedback
}

\author{
Han Jian Ning ${ }^{1}$ \\ School of Computer Science, Xi'an Shi You University ,Xi'an,Shannxi,710065,P,R,China
}

\begin{abstract}
Fingerprint classification has always been an important research direction in the field of intelligent recognition. Based on the method of fingerprint classifier integration, the backtracking feedback mechanism is introduced, and a fingerprint classification system with high recognition rate is designed. Through the use of 1000 fingerprint images in the fingerprint library to test, The system show the recognition results due to the current Kalle Karu, anli K.jain design of a variety of fingerprint recognition system. Through a series of experimental comparisons, it is proved that the fingerprint classification recognition system with the feedback mechanism has better ability of fingerprint recognition, and greatly reduces the error rate of system recognition.
\end{abstract}

\section{Introduction}

Using multi-classifier integration method can solve various complex problems such as pattern recognition. It is the rational integration of each single classifier, in order to play their respective advantages, learn from each other, improve the recognition rate of integrated system.

Feedback mechanism is not a new thing, all kinds of methods and concepts develop rapidly in many fields of research and application, and have been widely used, but it is rare to try to introduce feedback mechanism in pattern recognition

\section{Fingerprint Classification}

Everyone knows that each person's fingerprint has the unique characteristic of life, cannot repeat, the fingerprint recognition becomes the common method of the identification technology. At present, fingerprint recognition is still a focus problem in the field of pattern recognition with high recognition rate.

As an important research direction of automatic fingerprint recognition system, fingerprint recognition generally divides the fingerprint image into: arched, Zoo type, right ring type, sharp arch, scar type and vortex type six attribute parts. Because the fingerprint image is affected by a variety of complex factors, such as various noises, skin elasticity, roughness, acquisition technology, it is difficult to apply the fingerprint recognition by single automatic fingerprint classifier.

Integrating and drawing on the research methods of fingerprint classification problem, the paper introduces the vertex backtracking feedback mechanism, which reduces the recognition rate of fingerprint recognition error.

\section{System structure}

In view of the research problem, the system passes the following steps to determine the type of fingerprint image processed:

Including fingerprint image, image preprocessing, feature extraction, vector correction, main classifier, judgment device, Feedback link, auxiliary classifier. Described below:

\subsection{Image processing}

Image processing is mainly based on two methods of image segmentation and image enhancement.

\subsubsection{Image segmentation}

Image segmentation first through the segmentation of the fingerprint image of the cut, on the basis of as far as possible lossless fingerprint information generate a standard rectangular fingerprint image (i.e. standard fingerprint). This reduces the image data that is unrelated to the image processing and that can cause interference. The segmentation process can form a certain angle, which can reduce the deviation of the same fingerprint due to different rotation.

\subsubsection{Image increase}

Image segmentation adopts an improved algorithm, that is, using two-dimensional fast Fourier transform (FFT) to transform the information of fingerprint image into frequency domain; Based on this, a nonlinear transform

\footnotetext{
* Corresponding author: 1jnhan@126.com
} 
is used to enhance the available information ratio; Finally, the enhanced frequency domain information is converted to spatial information by inverse two-dimensional fast Fourier transform (IFFT).

The Nonlinear transformation formula is:

$$
\mathrm{U}_{\mathrm{jk}}+\mathrm{iV}_{\mathrm{jk}}=\left(\mathrm{X}_{\mathrm{jk}}^{2}+\mathrm{Y}_{\mathrm{jk}}^{2}\right) \operatorname{pow}\left(\mathrm{X}_{\mathrm{jk}}+\mathrm{Y}_{\mathrm{jk}}\right)
$$

Here, $\left(X_{j k}+Y_{j k}\right)$ represents the frequency domain information before the transformation, $\left(X_{j k}^{2}+Y_{j k}^{2}\right)$ pow represents the power spectral density information of the POW power, and $\mathrm{U}_{\mathrm{jk}}+\mathrm{iV}_{\mathrm{jk}}$ represents the transformed frequency domain information. The POW general initial value is 0.3

In fact, we want to enhance the information itself in the frequency domain, strong information is generally corresponding to the ridge and valley in the fingerprint map, more obvious, so the non-linear transformation naturally also strengthens the fingerprint image of useful information

\subsection{Feature extraction}

It mainly detects the direction of Ridge and Valley on each pixel of fingerprint image, and then produces an array of directions after a region draw, which can be used to record the basic features used in this system and to facilitate other processing. Based on the characteristics of the whole gray-scale change of fingerprint image, the local adaptive feature is converted into black-and-white fingerprint image by the domain value. Calculates the direction of Ridge and Valley on each pixel, then averages the direction, reduces the amount of data and noise, and obtains a basic feature set.

\subsection{Vector correction}

Vector correction is mainly used to reduce the errors that may occur when the array of similar directions is transformed. Usually different pattern of fingerprint direction array may exist in the ridge, Valley direction similar situation, so that the characteristics generated after processing caused by the transformation of large errors. Vector correction is used to smooth these errors.

\section{4 fingerprint classifier}

A variety of common fingerprint classification algorithms, which comes down to a typical voting Methods, intersection of decision regions, prediction by Top Choice combination, Dempster-shafer, wait.

\subsubsection{Main classifier}

The main classifier is constituted by probabilistic neural network (probabilistic neural network ,PNN). The input is a vector feature, which produces probability distributions in various categories, and the highest probability is the credibility of this classification. PNN consists of the following:
$\mathrm{N}=$ classification number (The previous six categories, with a value of 6 )

$\mathrm{M}_{\mathrm{j}}=$ Class I example number $(1 \leq \mathrm{i} \leq \mathrm{N})$

$\mathrm{X}_{\mathrm{j}}^{(\mathrm{i})}=$ Characteristic vectors for Class I J examples $\left(1 \leq \mathrm{j} \leq \mathrm{M}_{\mathrm{j}}\right)$

$\mathrm{W}=$ Feature vectors to classify

$\beta=$ smooth factor

$\alpha_{\mathrm{i}}=$ Class I activation amount

$\widetilde{\alpha}_{1}=$ Amount of activations after normalization

$\mathrm{h}=$ assume classification

$\mathrm{c}=$ the credibility

Calculation formula of PNN activation amount of Class I

$$
\begin{aligned}
& \alpha_{i}=\sum_{j=1}^{M_{i}} \exp \left(-\beta\left(w-x_{j}^{(i)}\right)^{t}\left(w-x_{j}^{(i)}\right)\right) \\
& \alpha_{h}, \quad \alpha_{j}(1 \leq \mathrm{i} \leq \mathrm{N}) \\
& \mathrm{c}=\widetilde{\alpha}_{h}=\alpha_{h} / \sum_{i=1}^{N} \alpha_{i}
\end{aligned}
$$

\subsubsection{Auxiliary classifier}

Auxiliary classifier for the main classifier of the Vortex class-aided classification, reduce the recognition error

\subsubsection{Judgment}

The judgment is combined with the classification results of the main and auxiliary classifiers, which set their respective advantages and enhance the recognition probability. If the main and auxiliary classifier output vortex type is consistent, the confidence level is 1 , the inconsistency confidence is 0.9 .

\section{Feedback mechanism}

In order to improve the recognition rate, the feedback mechanism is introduced to the classifier integration system, so that the system can adjust the recognition information automatically according to the feedback information, so as to achieve satisfactory results. According to the experiment, it can be observed that the main reason of high false recognition rate can be traced back to the fingerprint features extracted during the pretreatment stage, in which the main effect is more image noise, and of course, the reason of improper image segmentation, therefore, it is a good attempt to introduce the feedback mechanism into the image preprocessing:

If the credibility of the final judgment output is less than the setting of a certain class of domain value $\mathrm{T}_{\mathrm{j}} \quad\left(\mathrm{T}_{\mathrm{j}}\right.$ is the different fingerprint Class I, set the domain value $\mathrm{T}_{\mathrm{j}}$ ), the feedback to the image enhancement, increases the 
power of the POW in the transformation to achieve the effect of further filtering noise; If the output of the judgment device is greater than $T_{j}$, no feedback.

If the output of the judgment is still less than $T_{j}$ after multiple feedback (more than the set number), it may be the problem of image segmentation, which is to feedback the feedback mechanism to image segmentation and re-modify the segmentation parameters.

Comprehensive judgment device various possible output maximum, modify the segmentation parameters: that is, change the split rectangle parameters and rotation angle, to improve the extracted eigenvector parameter values, reduce the false.

Through feedback modification of the split parameters multiple times (to achieve the set number of times), the credibility of the output of the judgment is still less than $\mathrm{T}_{\mathrm{j}}$ words, no longer feedback, you can choose the maximum value of the output of the successive judgments the most final credibility, so as to determine the final fingerprint type.

The experimental results show that the effect of fingerprint recognition is improved by introducing the backtracking feedback mechanism, which greatly reduces the error rate of fingerprint recognition.

\section{Demonstration}

By testing the fingerprint in 1000 fingerprint libraries, it can be seen that the fingerprint error rate without back-feedback mechanism is $7.6 \%$, see table 1 , and the fingerprint error rate is $5.2 \%$ when the backtracking feedback mechanism is added. See table 2.

Table 1. Classification results (before feedback)

\begin{tabular}{|c|c|c|c|c|c|c|}
\hline \multicolumn{7}{|c|}{ Class } \\
\hline classification & $\begin{array}{c}\text { Arch } \\
\text { class }\end{array}$ & Zoo class & $\begin{array}{c}\text { Right ring } \\
\text { class }\end{array}$ & $\begin{array}{c}\text { scar } \\
\text { type }\end{array}$ & $\begin{array}{c}\text { sharp arch } \\
\text { class }\end{array}$ & $\begin{array}{c}\text { Vortex } \\
\text { class }\end{array}$ \\
\hline Arch class & $15(74,2)$ & $1(5,3)$ & $1(5,3)$ & $0(0,0)$ & $1(5,3)$ & $0(0,0)$ \\
\hline Zoo class & $0(0,0)$ & $261(95,0)$ & $2(1,4)$ & $0(0,0)$ & $2(1,5)$ & $6(2,7)$ \\
\hline $\begin{array}{c}\text { Right ring } \\
\text { class }\end{array}$ & $3(0,8)$ & $3(2,5)$ & $239(95,1)$ & $0(0,0)$ & $3(2,4)$ & $6(3,5)$ \\
\hline scar type & $0(0,0)$ & $0(0,0)$ & $0(0,0)$ & $0(0,0)$ & $0(0,0)$ & $1(103,0)$ \\
\hline sharp arch \\
class
\end{tabular}

Table 2. Classification results (after feedback)

\begin{tabular}{|l|l|l|l|l|l|l|}
\hline \multicolumn{7}{|c|}{ Class } \\
\hline classification & $\begin{array}{l}\text { Arch } \\
\text { class }\end{array}$ & Zoo class & $\begin{array}{l}\text { Right ring } \\
\text { class }\end{array}$ & $\begin{array}{l}\text { scar } \\
\text { type }\end{array}$ & $\begin{array}{l}\text { sharp } \\
\text { arch class }\end{array}$ & $\begin{array}{l}\text { Vortex } \\
\text { class }\end{array}$ \\
\hline Arch class & $17(90.5)$ & $0(0,0)$ & $1(5,3)$ & $0(0,0)$ & $1(5,3)$ & $0(0,0)$ \\
\hline Zoo class & $0(0,0)$ & $267(98,8)$ & $2(1,4)$ & $0(0,0)$ & $2(1,5)$ & $6(2,7)$ \\
\hline $\begin{array}{l}\text { Right ring } \\
\text { class }\end{array}$ & $2(0,8)$ & $2(2,5)$ & $242(95,5)$ & $0(0,0)$ & $2(2,4)$ & $6(3,5)$ \\
\hline scar type & $0(0,0)$ & $0(0,0)$ & $0(0,0)$ & $0(0,0)$ & $0(0,0)$ & $1(100,0)$ \\
\hline $\begin{array}{l}\text { sharp arch } \\
\text { class }\end{array}$ & $4(12,4)$ & $6(36,5)$ & $2(1,1)$ & $0(0,0)$ & $18(50,7)$ & $0(0,0)$ \\
\hline Vortex class & $0(0,0)$ & $7(4,7)$ & $7(1,8)$ & $0(0,0)$ & $0(0,0)$ & $410(96,9)$ \\
\hline
\end{tabular}

By introducing the backtracking feedback mechanism into the integrated fingerprint recognition system, it can obviously reduce the recognition error rate, improve the ability of the system to recognize the fingerprint, and can become a good attempt in the fingerprint identification direction. At present, there have been the United States FBI design fingerprint recognition system, has achieved good results, in contrast, the introduction of the retrospective feedback mechanism introduced by the integrated classification method and the recognition results in some aspects of better results, I believe that through the future continuous improvement, fingerprint recognition effect will be further improved

\section{References}

1. V.S. Srinivasasn and N. N. Murlthy, Detection of Singular Points in fingerprint images, Pattern Recognition 25(2),139-153(1992)

2. C.L. Wilson. Personal Communication. Luigi T. De Luca, Propulsion physics (EDP Sciences, Les , 2009)

3. B. Miller, Vital signs of identity, IEEE spectrum 31(2),22-33(1994)

4. Kalle Karu and Anli K.Jain,Fingerprint Classification Pattern, Vol 29,404(1996)

\section{Conclusion}

